VERSION OCTOBER 9, 2018: FM

Preprint typeset using $\mathrm{LAT}_{\mathrm{E}} \mathrm{X}$ style emulateapj v. 11/10/09

\title{
OPTICAL SPECTROSCOPIC OBSERVATIONS OF BLAZARS AND $\gamma$-RAY BLAZAR CANDIDATES IN THE SLOAN DIGITAL SKY SURVEY DATA RELEASE NINE
}

\author{
F. Massaro ${ }^{1}$, N. Masetti ${ }^{3}$, R. D'Abrusco ${ }^{4}$, A. Paggi $^{4}$, \& S. Funk ${ }^{2}$ \\ version October 9, 2018: fm
}

\begin{abstract}
We present an analysis of the optical spectra available in the Sloan Digital Sky survey data release nine (SDSS DR9) for the blazars listed in the ROMA-BZCAT and for the $\gamma$-ray blazar candidates selected according to their IR colors. First, we adopt a statistical approach based on MonteCarlo simulations to find the optical counterparts of the blazarslisted in the ROMA-BZCAT catalog. Then we crossmatched the SDSS spectroscopic catalog with our selected samples of blazars and $\gamma$-ray blazar candidates searching for those with optical spectra available to classify our blazar-like sources and, whenever possible, to confirm their redshifts. Our main objectives are determining the classification of uncertain blazars listed in the ROMA-BZCAT and discovering new gamma-ray blazars. For the ROMA-BZCAT sources we investigated a sample of 84 blazars confirming the classification for 20 of them and obtaining 18 new redshift estimates. For the $\gamma$-ray blazars, indicated as potential counterparts of unassociated Fermi sources or with uncertain nature, we established the blazar-like nature of 8 out the 27 sources analyzed and confirmed 14 classifications.
\end{abstract}

Subject headings: methods: statistical - galaxies: active - quasars: general - surveys - radiation mechanisms: non-thermal

\section{INTRODUCTION}

According to the well assessed unification scenario of the active galactic nuclei (AGN; e.g., Antonucci 1993; Urry \& Padovani 1995) blazars are radio loud sources, featuring compact radio cores combined with a "flat" radio spectra that extends from frequencies below $\sim 1 \mathrm{GHz}$ (e.g., Massaro et al. 2013a; Massaro et al. 2013b; (Nori et al. 2013) up to the submillimeter band (e.g., Giommi et al. 2012). They are characterized by a variable, non-thermal, continuum and exhibit a typical double bumped spectral energy distribution (SED), and represent the largest known population of $\gamma$-ray sources (e.g., Abdo et al. 2010a; Ackermann et al. 2011) proving the most relevant contribution to the extragalactic $\gamma$-ray background (e.g., Mukherjee et al. 1997; Abdo et al. 2010b).

Blazars are generally classified on the basis of their optical spectra and divided in two main classes: i) BL Lac objects, labeled as BZBs according to the nomenclature of the ROMA-BZCAT'5 (Massaro et al. 2009; Massaro et al. 2011a) when presenting featureless optical spectra and ii) flat spectrum radio quasars (hereinafter BZQs) having a typical quasar-like optical appearance but also featuring high and variable optical polarization. In particular, blazars are classified as BZB if the rest-frame equivalent width of their optical features is lower than $5 \AA$ (Stickel et al. 1991; Stoke et al. 1991;

\footnotetext{
${ }^{1}$ Yale Center for Astronomy and Astrophysics, Physics Department, Yale University, PO Box 208120, New Haven, CT 06520-8120, USA

2 SLAC National Laboratory and Kavli Institute for Particle Astrophysics and Cosmology, 2575 Sand Hill Road, Menlo Park, CA 94025, USA

${ }^{3}$ INAF - Istituto di Astrofisica Spaziale e Fisica Cosmica di Bologna, via Gobetti 101, 40129, Bologna, Italy

${ }^{4}$ Harvard - Smithsonian Astrophysical Observatory, 60 Garden Street, Cambridge, MA 02138, USA

5 http://www.asdc.asi.it/bzcat/
}

Laurent-Muehleisen et al. 1999; Landoni et al. 2013).

As recently discovered using the WISE all-sky survey (Wright et al. 2010), blazars show by peculiar infrared (IR) colors (Massaro et al. 2011b) mostly due to their non-thermal continuum that allowed to distinguish them from other classes of active galaxies (e.g., D'Abrusco et al. 2012; Massaro et al. 2012a). This IR property was also interpreted as due to the lack of observational signatures form a dusty torus in the case of BZBs (e.g., Plotkin et al. 2012).

The variable, non-thermal emission of both BZBs and BZQs, extending from radio up to TeV energies, is interpreted as arising from high-energy particles accelerated in a relativistic jet oriented along to the line of sight, whereas relativistic effects amplifies both their luminosity and the amplitude of their variability (Blandford \& Rees 1978; Giommi et al. 2013).

Recently, we searched for blazar-like objects as potential counterparts of the unidentified $\gamma$-ray sources (UGSs) observed with Fermi (Abdo et al. 2010a; Nolan et al. 2012) with several methods based on the IR colors alone (Massaro et al. 2012b; D'Abrusco et al. 2013) or combined with other multifrequency observations, as radio (Massaro et al. 2013d) or X-ray properties (Paggi et al. 2013). We also explored the use of low radio frequency observations (i.e., below $\sim 1 \mathrm{GHz}$ ) as an alternative possibility to find blazar-like counterparts (e.g., Massaro et al. 2013a; Nori et al. 2013) for the UGSs listed in the second Fermi-Large Area Telescope (LAT) catalog (2FGL, Nolan et al. 2012) in addition to other multifrequency analysis (e.g., Mirabal \& Halpern 2009; $\quad$ Ackermann et al. 2012; Cowperthwaite et al. 2013; Masetti et al. 2013). All these investigations provided several lists of gamma-ray blazar candidates that has to be confirmed and classified via optical spectroscopy.

Here we investigate the optical spectra of two 
blazar samples that lie in the footprint of the spectroscopic catalog of Sloan Digital Sky Survey data release 9 (SDSS DR9, Ahn et al. 2012). The first sample includes all the ROMA-BZCAT sources that have an uncertain classification, uncertain redshift estimates or have been classified as BL Lac candidates due to the lack of an optical spectrum in literature (Massaro et al. 2011a). The second sample lists all the $\gamma$-ray blazar candidates, that were identified as potential counterparts of UGSs in our previous analyses, and for which SDSS spectra are now available. This study is complementary to on going spectroscopic campaigns planned to investigate blazar optical properties (e.g., Sbarufatti et al. 2005; Sbarufatti et al. 2009; Plotkin et al. 2010; Paggi et al. 2014).

The paper is organized as follows. In Section 2 we present the statistical approach adopted to determine the optical counterparts of the ROMA-BZCAT sources in the SDSS DR9 catalog while in Section 3 we describe the samples considered in our analysis. In Section 4 the results of the spectroscopic analysis are illustrated while, finally, Section 5 is devoted to our summary and conclusions. For our numerical results, we use cgs units unless stated otherwise.

\section{SPATIAL ASSOCIATIONS}

The ROMA-BZCAT was mainly compiled on the basis of radio, optical and X-ray surveys and the blazar coordinates reported are not uniform. The positional accuracy is generally less than $<1^{\prime \prime}$ but it could reach $\sim 5^{\prime \prime}$, corresponding to the typical uncertainty on the radio positions of the NVSS, for those sources with radio flux densities close to the survey limit (Condon et al. 1998). Since the positional uncertainties for each source are not reported in the ROMA-BZCAT to identify the SDSS optical counterparts of the ROMA-BZCAT blazars we adopted the following a statistical approach.

First we computed the total number of ROMA-BZCAT blazars that lie within the footprint of the SDSS, corresponding to 1820 sources. For each blazar, we counted the total number of optical counterparts in the SDSS $N(R)$ present within circular regions of variable radius $R$ in the range between $0^{\prime \prime}$ and $10^{\prime \prime}$. To be conservative in our analysis, we only included in the $N(R)$ calculation SDSS sources having the flags: CLASS_OBJECT (i.e., mode) and CODE_MISC (i.e., clean) both equal to 16 .

We then created 100 mock realizations of the ROMABZCAT by shifting each blazar position in a random direction of the sky by a fixed length of $30^{\prime \prime}$. The shift used to create the mock ROMA-BZCAT catalogs were chosen not too distant from the original ROMA-BZCAT location and within the SDSS footprint. This guarantees to obtain fake catalogs with a sky distribution similar to the original ROMA-BZCAT and to crossmatch each fake catalog and the SDSS taking into account the local density distribution of the optical sources. The total number of blazars in each mock realization is also preserved being equal to that of the ROMA-BZCAT sources that lie in the SDSS footprint. For each mock realization of the ROMA-BZCAT we counted the number of associations with the SDSS occurring at angular separations $R$ smaller than $10^{\prime \prime}$. Then we computed the mean num-

${ }^{6}$ http://cas.sdss.org/dr7/sp/help/browser/browser.asp?n=PhotoObj\&t $=\mathrm{U}$

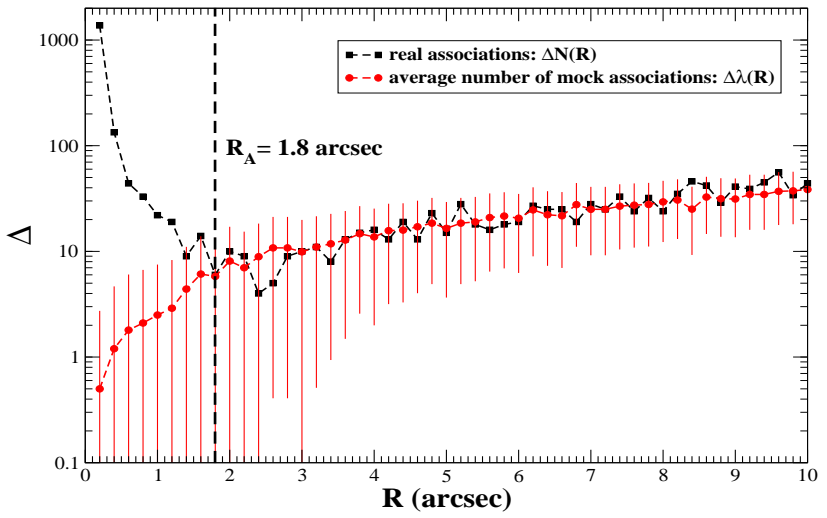

FIG. 1. - The values of $\Delta \lambda(R)$ (red circles) and $\Delta N(R)$ (black squares) as function of the angular separation $R$. We restricted the $\mathrm{x}$ axis to $R$ values below $10^{\prime \prime}$. Our choice of $R_{A}$ is marked by the vertical dashed line. It occurs at the first $R$ value for which $\Delta \lambda(R) \simeq \Delta N(R)$.

ber $\lambda(R)$ of these fake associations, averaged over the 100 mock ROMA-BZCAT catalogs, verifying that $\lambda(R)$ has a Poissonian distribution. Increasing the radius by $\Delta R=0^{\prime \prime} .2$, we also calculated the difference $\Delta \lambda(R)$ as:

$$
\Delta \lambda(R)=\lambda(R+\Delta R)-\lambda(R),
$$

In Figure 1 we show the comparison between $\Delta N(R)$ and $\Delta \lambda(R)$. For radii larger than $R_{A}=1^{\prime \prime} .8$ the $\Delta \lambda(R)$ curve superimposes that of $\Delta N(R)$ indicating that ROMA-BZCAT-SDSS cross-matches could occur by chance at angular separations larger than $R_{A}$. Thus we choose $1^{\prime \prime} .8$ as to the maximum angular separation between the ROMA-BZCAT and the SDSS position at which we consider the optical source a reliable counterpart of the blazar in the ROMA-BZCAT.

Finally the chance probability of spurious associations $p\left(R_{A}\right)$ was calculated as the ratio between the number of real associations $N\left(R_{A}\right)$ and the average of those found in the mock realizations of the ROMA-BZCAT $\lambda\left(R_{A}\right)$, corresponding to a value of $\sim 1 \%$ (see also Maselli et al. 2010; Massaro et al. 2011b; D'Abrusco et al. 2013, for additional details on $p\left(R_{A}\right)$ ).

\section{SAMPLE SELECTION}

We adopted the value of $R_{A}$ of $1^{\prime \prime} .8$ to search for the optical counterparts of the blazar-like sources in our two samples within the available spectra of the SDSS DR9 to confirm the source nature and whenever possible to estimate the redshift. Then we analyzed two samples of sources that lie in the footprint of the SDSS dr9 and with optical spectra available defined in the following.

1. The total number of ROMA-BZCAT sources having a optical counterpart in the SDSS DR9 spectroscopic catalog within $R_{A}$ is 219 . Among them there are 50 blazars with an uncertain redshift estimate and additional 34 sources classified as BL Lac candidates for which optical spectra were not available in literature while ROMA-BZCAT v4.1 was prepared. These 84 ROMA-BZCAT sources, 
with a unique correspondence in the SDSS DR9 spectroscopic catalog within $1^{\prime \prime} .8$, constitute our first sample investigated.

2. The second sample lists 15 blazar-like sources with uncertain classification and/or uncertain $z$ estimates included in the 2FGL (Nolan et al. 2012) and in the Second Fermi-LAT AGN Catalog (2LAC Ackermann et al. 2011) plus additional 12 sources identified as potential counterparts of UGSs according to their peculiar IR colors in our recent analyses (e.g., Massaro et al. 2012a; Massaro et al. 2012b; Massaro et al. 2013c). All these 27 sources also have a unique correspondence in the SDSS DR9 spectroscopic catalog within $1^{\prime \prime} .8$.

\section{RESULTS}

We visually inspected all the optical spectra available for the sources in our samples to avoid misleading classifications due to artifacts of the SDSS automatic analysis, and if necessary, for example to confirm a redshift estimate, we downloaded and analyzed the raw data.

We remark that for the BZB classification we adopted the criterion described in Laurent-Muehleisen et al. (1999), measuring the rest-frame equivalent widths of the emission and/or absorption lines whenever they are detectable above the continuum (see also the recent analyses performed by Sbarufatti et al. 2006; Landoni et al. 2013). In addition, we also adopted the criterion developed by Massaro et al. (2013c) to classify BL Lac object based on the SDSS (u-r) color, that supersedes the one based on the Ca H\&K break contrast originally introduced by Stoke et al. (1991). Thus, for each source, we computed the absorption corrected (ur) color equal to $(u-r)_{o b s}-0.81 * A_{r}$, where $A_{r}$ is the Galactic extinction in the $\mathrm{R}$ band, and we considered $\mathrm{BL}$ Lac objects only those sources with $(u-r)<1.4$ (see also Maselli et al. 2013). We assign a BZB classification only to sources having both a "featureless" spectra and the $(u-r)$ color lower than 1.4.

\subsection{ROMA-BZCAT blazars}

In the first sample of 84 ROMA-BZCAT blazars with SDSS DR9 spectra, we found that there are 3 BZQs, all associated to Fermi sources in the 2FGL and in the 2LAC (Nolan et al. 2012; Ackermann et al. 2011), with an uncertain $z$ estimate. Our analysis of their optical SDSS spectra allowed us to confirm both their nature and their redshifts. In addition to these BZQs, there are 47 sources out of the 84 listed in the first sample classified as BZB according to the ROMA-BZCAT but with an uncertain redshift estimate. We found that 9 of them have good SDSS spectra from which we obtained a $z$ measurement. Unfortunately none of these 9 is detected in the $\gamma$-rays.

The remaining 34 sources out of 84 objects in the first sample are indeed classified as BL Lac candidates, 5 of them being associated to Fermi sources in the 2LAC. We confirmed the BL Lac nature for 20 of them, including all the Fermi sources and providing a new redshift estimate for 6 . The remaining 14 sources were classified as: normal galaxies (8), type 2 Seyfert galaxies (5), according to the criteria described in Winkler (1992) plus
1 source having still an uncertain nature, mostly resembling a type 2 AGN.

All our results are summarized in Table2, where we report the ROMA-BZCAT and the SDSS names, together with the results of our analysis (i.e., classification and redshift estimates when possible) and their (u-r) colors. Blazars that are associated to Fermi sources are also indicated. Uncertain values of redshifts are indicated with a question mark (?); they are due to the poor signal to noise of few SDSS archival spectra or to the presence of only a single emission/absorption feature. Then, in Figure 2 we show the optical spectrum of one of the BL Lac classified from our analysis together with two cases of wrong classifications and a quasar.

\section{2. $\gamma$-ray blazar candidates}

The second sample of $\gamma$-ray blazar candidates selected according to our IR based procedures and having SDSS spectra available lists: 27 sources. Fifteen blazar-like sources were already present in the 2LAC but with uncertain classification or uncertain redshift estimates. Among them we found 7 having quasar-like optical spectra and being classified as BZQs, 2 also with new $z$ estimates, 7 BZBs including 2 sources with measured redshifts and 1 misclassified object: SDSS J122011.88+020342.2, associated with 2FGLJ1219.7+0201 that appears to be a Seyfert galaxy rather than BZQ.

For 2FGLJ1023.6+3947, associated to the SDSS J102333.50+395312.7 source, the we obtained a redshift of 1.3328 instead of 1.254 reported in the $2 \mathrm{LAC}$ and for the BL Lac object SDSS J110021.05+401928.0 counterpart of 2FGLJ1100.9+4014, we were not able to find any optical feature to confirm the 2LAC redshift of 0.225 . Among these 15 sources there is SDSS J222329.57+010226.6, associated to the AGN of uncertain type 2FGLJ2223.4+0104 and selected in Cowperthwaite et al. (2013) as a $\gamma$-ray blazar candidate that we confirmed as a BL Lac at unknown redshift.

The remaining 12 sources were all selected as $\gamma$ ray blazar candidates in our previous analyses of their IR colors (Massaro et al. 2012b; Massaro et al. 2013c; Massaro et al. 2013a; Paggi et al. 2013). We found that 3 sources, all with new $z$ estimates, out of 12 have a quasar like spectrum, similar to those of the BZQs, plus one uncertain due to noisy SDSS spectrum (i.e., SDSS J015852.77+010132.8). Then there are 4 confirmed BL Lac objects while the remaining 4 sources are indeed contaminants of the association methods ( 1 star and 4 Seyfert galaxies).

All these results are reported in Table 3 in the same order as discussed above, where we also indicate the previous classification of each source.

\section{SUMMARY AND CONCLUSIONS}

We performed an analysis of the archival optical spectra present in the SDSS DR9 (Ahn et al. 2012) for two selected samples of blazars and $\gamma$-ray blazar candidates to confirm their nature and whenever possible to estimate their redshifts.

First, we adopted a statical approach to find the the SDSS optical cross-matches of the sources in our sample. Then, we analyzed a first sample of 84 blazars listed in the ROMA-BZCAT as BL Lac candidates or 
as BL Lac objects and flat spectrum radio quasars with uncertain redshift estimates and a second sample of $27 \gamma$-ray blazar candidates selected according to their peculiar IR colors or with uncertain classification (e.g., D'Abrusco et al. 2013; Massaro et al. 2013c; Massaro et al. 2013d, and references therein).

On the basis of the SDSS spectra, we confirmed the redshifts for 3 flat spectrum radio quasars (all $\gamma$-ray sources detected by Fermi) and measured the $z$ for 9 additional BL Lacs investigated. Then, we have been able to classify 34 BL Lac candidates listed in the ROMABZCAT, 20 of them appearing as BL Lac objects, providing new $z$ estimates for 6 BL Lac objects. These spectroscopic information, even if available for a small fraction of the whole ROMA-BZCAT catalog will be essential to refine its future releases as well as those of the Fermi catalogs.

For the second sample listing $27 \gamma$-ray blazar candidates we found a total of 11 BZBs ( 2 with new $z$ measurements) and 11 BZQs having new redshift estimates for 2 of them. The remaining 5 sources did not appear to have the typical blazar-like optical spectrum. All our results are summarized in Table 4 .

We thank our anonymous referee for many helpful comments which greatly improved this manuscript. We are grateful to our colleague E. Palazzi that helped us in the data reduction of some SDSS spectra. The work is supported by the NASA grants NNX12AO97G and NNX13AP20G. Part of this work is based on archival data, software or on-line services provided by the ASI Science Data Center. This research has made use of data obtained from the high-energy Astrophysics Science Archive Research Center (HEASARC) provided by NASA's Goddard Space Flight Center; the SIMBAD database operated at CDS, Strasbourg, France; the
NASA/IPAC Extragalactic Database (NED) operated by the Jet Propulsion Laboratory, California Institute of Technology, under contract with the National Aeronautics and Space Administration. This publication makes use of data products from the Wide-field Infrared Survey Explorer, which is a joint project of the University of California, Los Angeles, and the Jet Propulsion Laboratory/California Institute of Technology, funded by the National Aeronautics and Space Administration. TOPCAT7 (Taylor 2005) for the preparation and manipulation of the tabular data and the images. Funding for the SDSS and SDSS-II has been provided by the Alfred P. Sloan Foundation, the Participating Institutions, the National Science Foundation, the U.S. Department of Energy, the National Aeronautics and Space Administration, the Japanese Monbukagakusho, the Max Planck Society, and the Higher Education Funding Council for England. The SDSS Web Site is http://www.sdss.org/ The SDSS is managed by the Astrophysical Research Consortium for the Participating Institutions. The Participating Institutions are the American Museum of Natural History, Astrophysical Institute Potsdam, University of Basel, University of Cambridge, Case Western Reserve University, University of Chicago, Drexel University, Fermilab, the Institute for Advanced Study, the Japan Participation Group, Johns Hopkins University, the Joint Institute for Nuclear Astrophysics, the Kavli Institute for Particle Astrophysics and Cosmology, the Korean Scientist Group, the Chinese Academy of Sciences (LAMOST), Los Alamos National Laboratory, the Max-Planck-Institute for Astronomy (MPIA), the MaxPlanck-Institute for Astrophysics (MPA), New Mexico State University, Ohio State University, University of Pittsburgh, University of Portsmouth, Princeton University, the United States Naval Observatory, and the University of Washington.

\section{REFERENCES}

Abdo, A. A. et al. 2010a ApJS 188405

Abdo, A. A. et al. 2010b ApJ, 720, 435

Ackermann, M. et al. 2011a ApJ, 743, 171

Ackermann, M. et al. 2012 ApJ, 753, 83

Ahn et al. 2012, ApJS, 203, 21

Antonucci, R. 1993 ARA\&A, 31, 473

Blandford, R.D. \& Rees, M. J. 1978, in Proc. Pittsburgh Conf. on BL Lac objects, (Pittsburgh, PA: Univ. Pittsburgh), 328

Cowperthwaite, P. S. et al. 2013 AJ, 146, 110

Condon, J. J. et al. 1998, AJ, 115, 1693

D'Abrusco, R. et al. 2012 ApJ, 748, 68

D'Abrusco, R. et al. 2013 ApJS, 206, 12

Giommi, P. et al. 2012 A\&A, 541A, 160

Giommi et al. 2013 MNRAS, 431, 1914

Landoni, M. et al. 2013 AJ, 145, 114

Laurent-Muehleisen, S.A. et al., 1999, ApJ, 525, 127

Maselli, A. et al. 2010 A\&A, 512A, 74

Maselli, A. et al. 2013 ApJS, 206, 17

Masetti, N. et al. 2013 A\&A, 559, A58

Massaro, E. et al. 2009 A\&A, 495, 691

Massaro, E. et al. 2011a "Multifrequency Catalogue of Blazars

(3rd Edition)", ARACNE Editrice, Rome, Italy

Massaro, F. et al. 2011b ApJ, 740L, 48

Massaro, F. et al. 2012a ApJ, 750, 138
Massaro, F. et al. 2012b ApJ, 752, 61

Massaro, E. et al. 2012 MNRAS, 422, 2322

Massaro, F. et al. 2013a ApJS, 207, 4

Massaro, F. et al. 2013b ApJS, 208, 15

Massaro, F. et al. 2013c ApJS, 206, 13

Massaro, F. et al. 2013d ApJS, 209, 10

Massaro, F. et al. 2013e ApJS, 207, 16

Mirabal, N. 2009 ApJ, 701, 129

Mukherjee, R. et al., 1997 ApJ, 490, 116

Nolan et al. 2012 ApJS, 199, 31

Nori, M. et al. 2014 ApJS, 212, 3

Paggi, A. et al. 2013 ApJS, 209, 9

Paggi, A. et al. 2014 AJ, 147, 112

Plotkin, R. M. 2010 AJ, 139, 390

Plotkin, R. M. et al. 2012 ApJ, 745L, 27

Sbarufatti et al. 2005, AJ, 129, 559

Sbarufatti et al. 2006, AJ, 132, 1

Sbarufatti, B. et al. 2009 AJ, 137, 337

Stickel, M. et al. 1991 ApJ, 374, 431

Stocke et al. 1991, ApJS, 76, 813

Taylor, M. B. 2005, ASP Conf. Ser., 347, 29

Urry, C. M., \& Padovani, P. 1995, PASP, 107, 803

Winkler H. 1992 MNRAS, 257, 677

Wright, E. L. et al. 2010 AJ, 140, 1868

${ }^{7}$ http://www.star.bris.ac.uk/ mbt/topcat/ 
SDSS J103220.28+030949.2

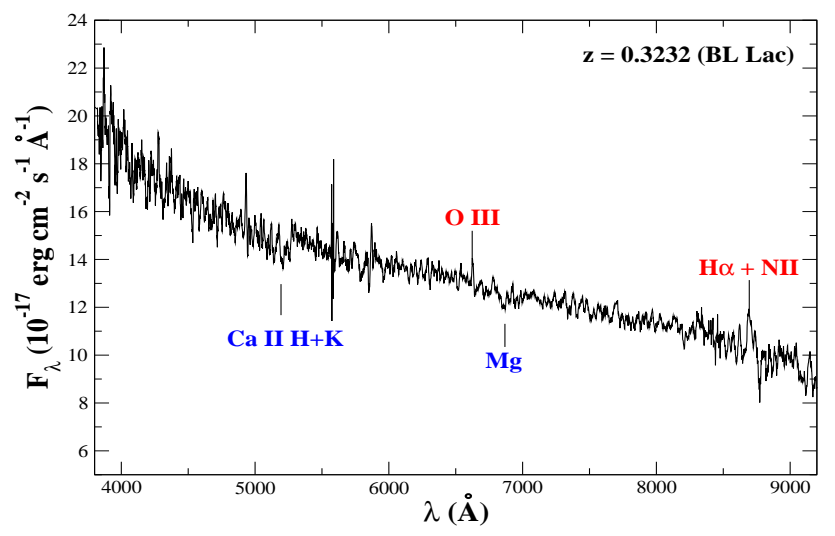

SDSS J122604.12+260427.9

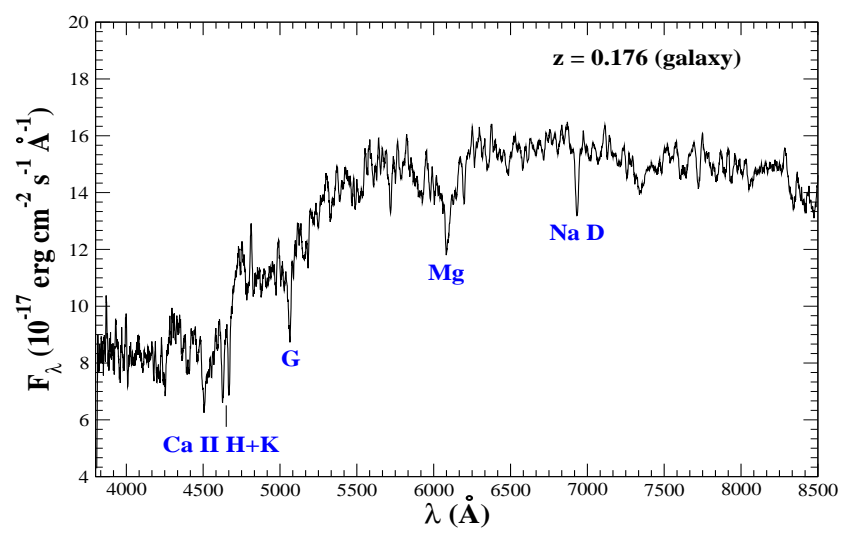

SDSS J002608.37-000547.0

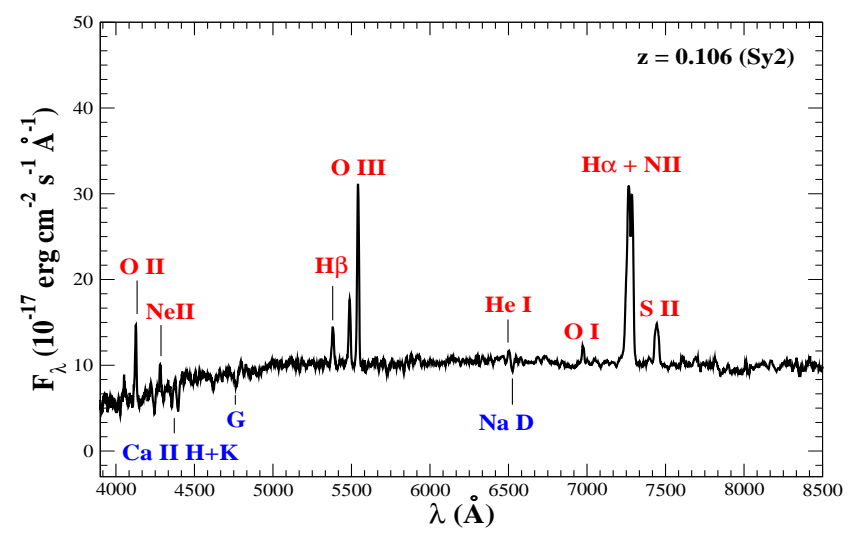

SDSS J132700.86+221050.1

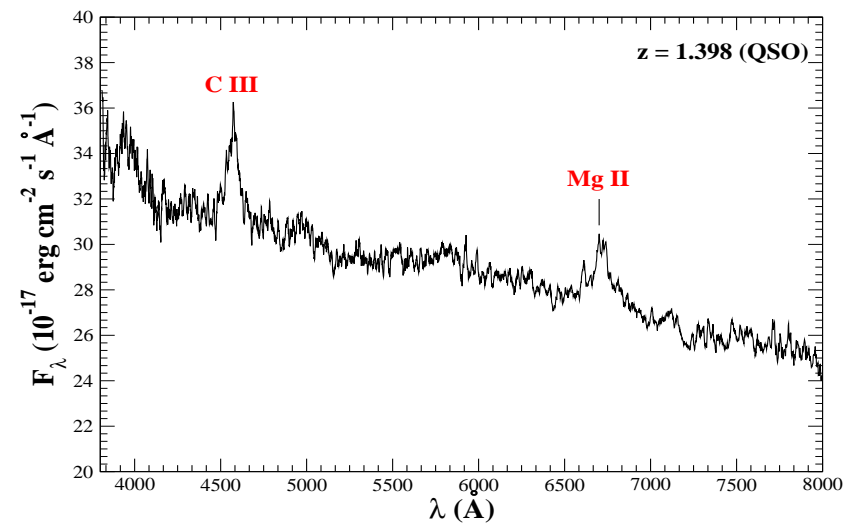

FIG. 2.- Upper left panel) The BL Lac object SDSSJ103220.28+030949.2 at redshift 0.3232. Upper right panel) The Seyfert 2 galaxy SDSSJ002608.37-000547.0 previously misclassified as a BL Lac candidate Lower left panel) The normal galaxy SDSSJ122604.12+260427.9 spectrum at redshift $\mathrm{z}=0.176$. Lower right panel) The $\mathrm{z}=1.398$ quasar SDSSJ132700.86+221050.1. The man in spectral emission and/or absorption features are marked in each figure. 
TABLE 1

Association of ROMA-BZCAT SOURCES.

\begin{tabular}{|c|c|c|c|c|c|c|}
\hline $\begin{array}{l}\text { BZCAT } \\
\text { name }\end{array}$ & $\begin{array}{l}\text { SDSS } \\
\text { counterpart }\end{array}$ & $\begin{array}{l}\text { BZCAT } \\
\text { class }\end{array}$ & $\begin{array}{l}\text { SDSS } \\
\text { class }\end{array}$ & $\begin{array}{l}\text { BZCAT } \\
\text { redshift }\end{array}$ & $\begin{array}{l}\text { SDSS } \\
\text { redshift }\end{array}$ & $u-r$ \\
\hline BZQJ0310+3814* & J031049.87+381453.8 & FSRQ & QSO & $0.816 ?$ & 0.816 & 1.02 \\
\hline BZQJ0830+2410* & J083052.08+241059.8 & FSRQ & QSO & $0.939 ?$ & 0.939 & 0.42 \\
\hline BZQJ1327+2210* & $\mathrm{J} 132700.86+221050.1$ & FSRQ & QSO & $1.4 ?$ & 1.398 & 0.44 \\
\hline BZBJ0001-0011 & J000121.46-001140.3 & BL Lac & BL Lac & $0.462 ?$ & 0.462 & 0.67 \\
\hline BZBJ0100-0055 & J010058.19-005547.7 & BL Lac & BL Lac & $0.67>?$ & ? & 0.98 \\
\hline BZBJ0127-0821 & J012716.31-082128.8 & BL Lac & BL Lac & $0.36 ?$ & $0.75 ?$ & 1.06 \\
\hline BZBJ0141-0928* & J014125.83-092843.7 & BL Lac & BL Lac & $0.73 ?$ & $0.03 ?$ & 0.96 \\
\hline BZBJ0731+2804 & J073152.73+280432.9 & BL Lac & BL Lac & $0.25 ?$ & 0.248 & 1.2 \\
\hline BZBJ0755+3726 & J075523.11+372618.7 & BL Lac & BL Lac & $0.606 ?$ & ? & 0.9 \\
\hline BZBJ0801+1336 & J080115.01+133642.2 & BL Lac & BL Lac & $1.042 ?$ & ? & 0.77 \\
\hline BZBJ0818+4222* & J081815.99+422245.4 & BL Lac & BL Lac & $0.53 ? ?$ & ? & 0.98 \\
\hline BZBJ0823+2223 & $\mathrm{J} 082324.75+222303.2$ & BL Lac & BL Lac & $0.951 ?$ & ? & 0.87 \\
\hline BZBJ0840+3440 & J084013.03+344026.9 & BL Lac & BL Lac & $0.451 ?$ & ? & 1.07 \\
\hline BZBJ0856+2057* & J085639.74+205743.3 & BL Lac & BL Lac & $0.18 ?$ & $0.2 ?$ & 0.83 \\
\hline BZBJ0926+5411 & J092638.87+541126.5 & BL Lac & BL Lac & $0.841 ?$ & $0.8 ?$ & 0.81 \\
\hline BZBJ $0940+2603$ & J094014.72+260330.0 & BL Lac & BL Lac & $0.498 ? ?$ & ? & 1.11 \\
\hline BZBJ0951+0102 & $\mathrm{J} 095127.81+010210.2$ & BL Lac & BL Lac & $0.502 ?$ & ? & 0.75 \\
\hline BZBJ1006+3454 & $\mathrm{J} 100656.46+345445.1$ & BL Lac & BL Lac & $0.612 ?$ & ? & 0.49 \\
\hline BZBJ1012+0630* & $\mathrm{J} 101213.34+063057.1$ & BL Lac & BL Lac & $0.518 ?$ & ? & 0.69 \\
\hline BZBJ $1031+5053^{*}$ & $\mathrm{~J} 103118.51+505335.8$ & BL Lac & BL Lac & $0.361 ? ?$ & ? & 0.45 \\
\hline BZBJ1032+0309 & $\mathrm{J} 103220.28+030949.2$ & BL Lac & BL Lac & $0.323 ?$ & 0.3232 & 0.64 \\
\hline BZBJ $1100+4019^{*}$ & $\mathrm{~J} 110021.05+401928.0$ & BL Lac & BL Lac & $0.225 ?$ & ? & -0.02 \\
\hline BZBJ $1107+5010$ & $\mathrm{~J} 110704.78+501037.9$ & BL Lac & BL Lac & $0.706 ?$ & 0.7062 & 0.99 \\
\hline BZBJ $1117+2548$ & $\mathrm{~J} 111740.39+254846.5$ & BL Lac & BL Lac & $0.36 ?$ & ? & 0.63 \\
\hline BZBJ $1120+4212^{*}$ & $\mathrm{~J} 112048.06+421212.4$ & BL Lac & BL Lac & $0.124 ? ?$ & ? & 0.34 \\
\hline BZBJ $1132+0034^{*}$ & $\mathrm{~J} 113245.62+003427.7$ & BL Lac & BL Lac & $1.223 ? ?$ & ? & 0.89 \\
\hline BZBJ1136+1601 & $\mathrm{J} 113617.53+160152.2$ & BL Lac & BL Lac & $0.574 ?$ & 0.5734 & 0.8 \\
\hline BZBJ $1138+4113$ & $\mathrm{~J} 113812.15+411352.8$ & BL Lac & BL Lac & $0.574 ?$ & $0.5740 ?$ & 1.1 \\
\hline BZBJ1211+2242 & $\mathrm{J} 121158.63+224232.9$ & BL Lac & BL Lac & $0.455 ?$ & 0.4527 & 0.53 \\
\hline BZBJ $1219+0446$ & $\mathrm{~J} 121944.97+044622.4$ & BL Lac & BL Lac & $0.489 ?$ & ? & 0.72 \\
\hline BZBJ $1231+2847^{*}$ & $\mathrm{~J} 123143.57+284749.7$ & BL Lac & BL Lac & $0.236 ?$ & ? & 0.68 \\
\hline BZBJ $1237+3020$ & $\mathrm{~J} 123705.61+302005.1$ & BL Lac & BL Lac & ? & ? & 0.36 \\
\hline BZBJ $1238+4431$ & $\mathrm{~J} 123826.01+443137.1$ & BL Lac & BL Lac & $0.312 ?$ & 0.3121 & 1.34 \\
\hline BZBJ $1239+4132$ & $\mathrm{~J} 123922.73+413251.4$ & BL Lac & BL Lac & $0.16 ? ?$ & ? & 0.53 \\
\hline BZBJ $1247+4423$ & $\mathrm{~J} 124700.72+442318.8$ & BL Lac & BL Lac & $0.6 ?$ & $0.73 ?$ & 0.51 \\
\hline BZBJ $1255+3848$ & $\mathrm{~J} 125555.40+384811.3$ & BL Lac & BL Lac & $0.559 ?$ & ? & 0.81 \\
\hline BZBJ $1328+1145$ & $\mathrm{~J} 132833.56+114520.5$ & BL Lac & BL Lac & $0.49 ?$ & ? & -0.06 \\
\hline BZBJ1401+3611 & $\mathrm{J} 140138.72+361121.9$ & BL Lac & BL Lac & $0.507 ?$ & $0.5064 ?$ & 1.21 \\
\hline BZBJ $1404+0402^{*}$ & $\mathrm{~J} 140450.90+040202.1$ & BL Lac & BL Lac & $0.344 ? ?$ & ? & 0.45 \\
\hline BZBJ $1423+1412$ & $\mathrm{~J} 142330.67+141247.9$ & BL Lac & BL Lac & $0.769 ? ?$ & $0.7687 ?$ & 0.6 \\
\hline BZBJ $1436+4129$ & $\mathrm{~J} 143627.16+412932.3$ & BL Lac & BL Lac & $0.404 ?$ & ? & 2.74 \\
\hline BZBJ $1436+5639^{*}$ & $\mathrm{~J} 143657.72+563924.8$ & BL Lac & BL Lac & $0.15 ?$ & ? & 0.47 \\
\hline BZBJ $1443+2515$ & $\mathrm{~J} 144334.40+251558.2$ & BL Lac & BL Lac & $0.529 ? ?$ & 0.5295 & 0.8 \\
\hline BZBJ $1456+5048$ & $\mathrm{~J} 145603.64+504825.9$ & BL Lac & BL Lac & $0.479 ?$ & ? & 0.52 \\
\hline BZBJ $1506+0814^{*}$ & $\mathrm{~J} 150644.47+081400.6$ & BL Lac & BL Lac & $0.376 ?$ & ? & 0.52 \\
\hline BZBJ $1553+0601$ & $\mathrm{~J} 155331.06+060143.8$ & BL Lac & BL Lac & $0.619 ?$ & 0.6189 & 0.98 \\
\hline BZBJ1603+1105 & $\mathrm{J} 160341.93+110548.7$ & BL Lac & BL Lac & $0.143 ? ?$ & ? & 0.94 \\
\hline BZBJ $1623+2841$ & $\mathrm{~J} 162332.25+284128.7$ & BL Lac & BL Lac & $0.377 ?$ & ? & 0.46 \\
\hline BZBJ $1652+3632$ & J165248.44+363212.5 & BL Lac & BL Lac & $0.648 ?$ & $0.6470 ?$ & 0.96 \\
\hline BZBJ $1701+3954$ & $\mathrm{~J} 170124.63+395437.0$ & BL Lac & BL Lac & $0.507 ?$ & ? & 0.27 \\
\hline
\end{tabular}

Col. (1) ROMA-BZCAT name.

optical counterpart.

Col. (3) ROMA-BZCAT classification.

encosic classification based on analysis.

Col. (5) Redshift estimate reported in the ROMA-BZCAT. Question mark indicates uncertain estimates.

Col. (6) Redshift estima
Col. (7) SDSS u-r color. 
TABLE 2

Association of ROMA-BZCAT SOURCES.

\begin{tabular}{|c|c|c|c|c|c|c|c|}
\hline $\begin{array}{l}\text { BZCAT } \\
\text { name }\end{array}$ & $\begin{array}{l}\text { SDSS } \\
\text { counterpart }\end{array}$ & $\begin{array}{l}\text { BZCAT } \\
\text { class }\end{array}$ & $\begin{array}{l}\text { SDSS } \\
\text { class }\end{array}$ & $\begin{array}{l}\text { BZCAT } \\
\text { redshift } \\
\end{array}$ & $\begin{array}{c}\text { SDSS } \\
\text { redshift }\end{array}$ & $\mathrm{u}-\mathrm{r}$ & $\begin{array}{c}\text { Classification } \\
\text { flag } \\
\end{array}$ \\
\hline BZBJ0026-0005 & J002608.37-000547.0 & BL Lac Can. & Sy 2 & 0.107 & 0.106 & 2.16 & no \\
\hline BZBJ0109+1816* & J010908.17+181607.5 & BL Lac Can. & BL Lac & 0.145 & ? & 0.7 & yes \\
\hline BZBJ0253-0124 & J025315.60-012405.3 & BL Lac Can. & BL Lac & ? & ? & 0.67 & yes \\
\hline BZBJ0754+4823* & J075445.66+482350.7 & BL Lac Can. & BL Lac & ? & ? & 0.93 & yes \\
\hline BZBJ0814+0856 & J081421.66+085706.1 & BL Lac Can. & galaxy & $0.23 ? ?$ & 0.24 & 7.33 & no \\
\hline BZBJ0829+1754 & J082904.82+175415.8 & BL Lac Can. & Sy 2 & 0.089 & 0.0895 & 2.12 & no \\
\hline BZBJ0831+5400 & J083100.36+540023.2 & BL Lac Can. & $\mathrm{Sy} 2$ & ? & 0.0617 & 3.06 & no \\
\hline BZBJ0839+4015 & J083903.08+401545.6 & BL Lac Can. & galaxy & 0.194 & 0.1941 & 2.43 & no \\
\hline BZBJ0905+4705 & J090536.44+470546.3 & BL Lac Can. & type2 2 & 0.174 & 0.1736 & 2.33 & no \\
\hline BZBJ0912+4235 & J091227.22+423545.1 & BL Lac Can. & galaxy & 0.266 & 0.2662 & 4.0 & no \\
\hline BZBJ0933+0003 & J093310.57+000323.5 & BL Lac Can. & BL Lac & ? & ? & 0.45 & yes \\
\hline BZBJ0944+5557 & J094441.47+555752.9 & BL Lac Can. & BL Lac & ? & ? & 0.93 & yes \\
\hline BZBJ $1007+5023$ & $\mathrm{~J} 100710.44+502356.4$ & BL Lac Can. & Sy 2 & 0.133 & 0.1326 & 1.87 & no \\
\hline BZBJ $1057+2303$ & $\mathrm{~J} 105723.09+230318.7$ & BL Lac Can. & BL Lac & 0.379 & 0.3782 & 1.07 & yes \\
\hline BZBJ1058+2817 & $\mathrm{J} 105829.89+281746.3$ & BL Lac Can. & BL Lac & ? & $0.4793 ?$ & 0.89 & yes \\
\hline BZBJ $1100+4210$ & $\mathrm{~J} 110020.99+421053.1$ & BL Lac Can. & galaxy & 0.323 & 0.3229 & 1.75 & no \\
\hline BZBJ1110+3539 & $\mathrm{J} 111056.83+353907.2$ & BL Lac Can. & BL Lac & ? & $0.61 ?$ & 0.72 & yes \\
\hline BZBJ1111+3452 & $\mathrm{J} 111130.90+345203.2$ & BL Lac Can. & BL Lac & 0.212 & ? & 0.42 & yes \\
\hline BZBJ1152+2837 & $\mathrm{J} 115210.70+283721.3$ & BL Lac Can. & BL Lac & ? & 0.4412 & 1.17 & yes \\
\hline BZBJ1153+3823 & $\mathrm{J} 115210.70+283721.3$ & BL Lac Can. & $\mathrm{Sy} 2$ & ? & 0.4098 & 0.99 & no \\
\hline BZBJ $1224+2239$ & $\mathrm{~J} 122401.03+223939.5$ & BL Lac Can. & BL Lac & ? & 0.4821 & 0.88 & yes \\
\hline BZBJ1226+2604 & $\mathrm{J} 122604.12+260427.9$ & BL Lac Can. & galaxy & 0.176 & 0.1761 & 2.0 & no \\
\hline BZBJ $1243+3627^{*}$ & $\mathrm{~J} 124312.73+362743.9$ & BL Lac Can. & BL Lac & ? & ? & 0.45 & yes \\
\hline BZBJ $1253+3826$ & $\mathrm{~J} 125300.95+382625.7$ & BL Lac Can. & BL Lac & 0.372 & 0.3707 & 1.08 & yes \\
\hline BZBJ $1311+0853$ & $\mathrm{~J} 131155.76+085340.9$ & BL Lac Can. & BL Lac & 0.469 & 0.4694 & 0.85 & yes \\
\hline BZBJ $1314+2348^{*}$ & $\mathrm{~J} 131443.80+234826.7$ & BL Lac Can. & BL Lac & ? & $0.15 ?$ & 0.72 & yes \\
\hline BZBJ1341+3716 & $\mathrm{J} 134138.66+371644.8$ & BL Lac Can. & galaxy & 0.17 & 0.1745 & 2.93 & no \\
\hline BZBJ $1404+2701$ & $\mathrm{~J} 140436.82+270141.0$ & BL Lac Can. & galaxy & 0.136 & 0.1383 & 2.67 & no \\
\hline BZBJ $1410+2820 *$ & $\mathrm{~J} 141029.56+282055.6$ & BL Lac Can. & BL Lac & $?$ & $?$ & 0.58 & yes \\
\hline BZBJ $1426+2415$ & $\mathrm{~J} 142645.52+241523.0$ & BL Lac Can. & BL Lac & $0.055 ? ?$ & $0.36 ?$ & 0.4 & yes \\
\hline BZBJ $1437+4717$ & $\mathrm{~J} 143716.14+471726.3$ & BL Lac Can. & BL Lac & ? & ? & 0.68 & yes \\
\hline BZBJ2129+0035 & $\mathrm{J} 212940.67+003527.4$ & BL Lac Can. & BL Lac & 0.425 & 0.4264 & -0.1 & yes \\
\hline BZBJ $2227+0037$ & $\mathrm{~J} 222758.13+003705.4$ & BL Lac Can. & BL Lac & ? & ? & 0.76 & yes \\
\hline BZBJ2319-0116 & J231952.83-011626.8 & BL Lac Can. & galaxy & ? & 0.2835 & 1.72 & no \\
\hline
\end{tabular}

Col. (1) ROMA-BZCAT name.
Col. (2) SDSS name of the optical counterpart.

Col. (2) SDSS name of the optical counte
Col. (3) ROMA-BZCAT classification.

Col. (4) SDSS spectroscopic classification based on our analysis.

Col. (6) Redshift estimate derived from our analysis. Question mark indicates uncertain estimates.

Col. (7) u-r color

Col. (8) Classification flag: (yes) marks sources that have been classified on the basis of our analysis.

TABLE 3

Association of $\gamma$-RAY Blazar candidates (00-24 HH).

\begin{tabular}{|c|c|c|c|c|c|}
\hline $\begin{array}{l}\text { Source } \\
\text { name }\end{array}$ & $\begin{array}{l}\text { SDSS } \\
\text { counterpart }\end{array}$ & $\begin{array}{l}\text { SDSS } \\
\text { class }\end{array}$ & $\begin{array}{l}\text { SDSS } \\
\text { redshift }\end{array}$ & $u-r$ & $\begin{array}{c}\text { Classification } \\
\text { flag }\end{array}$ \\
\hline 2FGLJ0323.6-0108 & J032343.62-011146.1 & BL Lac & $?$ & 0.58 & yes \\
\hline 2 FGLJ0924.0+2819 & J092351.52+281525.1 & QSO & 0.7442 & 0.57 & yes \\
\hline 2 FGLJ0950.1+4554 & J095011.82+455320.0 & BL Lac & 0.3994 & 0.99 & yes \\
\hline 2 FGLJ1017.0+3531 & J101810.97+354239.4 & QSO & 1.2280 & 0.42 & yes \\
\hline 2FGLJ1023.6+3947 & $\mathrm{J} 102333.50+395312.7$ & QSO & 1.3328 & 0.23 & yes \\
\hline 2FGLJ1100.9+4014 & $\mathrm{J} 110021.05+401928.0$ & BL Lac & ? & 0.43 & yes \\
\hline 2FGLJ1219.7+0201 & $\mathrm{J} 122011.88+020342.2$ & Sy 1.8 & 0.2402 & -0.02 & no \\
\hline 2FGLJ1222.4+0413 & $\mathrm{J} 122222.54+041315.7$ & QSO & 0.9642 & 0.44 & yes \\
\hline 2 FGLJ1301.6+3331 & $\mathrm{J} 130129.15+333700.3$ & QSO & 1.0084 & 0.75 & yes \\
\hline 2FGLJ1310.9+0036 & $\mathrm{J} 131106.47+003510.0$ & BL Lac & ? & 0.62 & yes \\
\hline 2FGLJ1351.4+1115 & $\mathrm{J} 135120.84+111453.0$ & BL Lac & ? & 0.56 & yes \\
\hline 2 FGLJ1332.7+4725 & J133245.24+472222.6 & QSO & 0.6687 & 0.74 & yes \\
\hline 2 FGLJ1442.0+4352 & $\mathrm{J} 144207.15+434836.7$ & BL Lac & ? & 0.78 & yes \\
\hline 2 FGLJ1522.0+4348 & $\mathrm{J} 152149.61+433639.2$ & QSO & 2.1677 & 0.35 & yes \\
\hline 2 FGLJ $2223.4+0104^{+}$ & $\mathrm{J} 222329.57+010226.6$ & BL Lac & $0.29 ?$ & 0.49 & yes \\
\hline 2FGLJ0158.4+0107 & J015852.77+010132.8 & QSO? & $1.61 ?$ & 0.95 & yes \\
\hline 2 FGLJ0440.5+2554 & J043947.48+260140.8 & star & 0 . & -1.56 & no \\
\hline 2 FGLJ0823.0+4041 & J082257.55+404149.7 & QSO & 0.8655 & 0.58 & yes \\
\hline 1FGLJ0835.4+0936 & J083543.21+093717.9 & BL Lac & $0.35 ?$ & 0.79 & yes \\
\hline 2FGLJ0844.9+6214 & J084406.82+621458.4 & Sy 1.9 & 0.1208 & 3.13 & no \\
\hline 2 FGLJ1129.5+3758 & J112903.24+375656.7 & BL Lac & ? & 1.14 & yes \\
\hline 2FGLJ1209.6+4121 & J120922.78+411941.3 & BL Lac & ? & 0.75 & yes \\
\hline $1 \mathrm{FGLJ} 1422.7+3743$ & $\mathrm{~J} 142304.61+373730.5$ & BL Lac & ? & 0.88 & yes \\
\hline 2FGLJ1612.0+1403 & $\mathrm{J} 161118.10+140328.7$ & QSO & 0.5855 & 0.15 & yes \\
\hline 2 FGLJ1614.8+4703 & $\mathrm{J} 161541.21+471111.7$ & Sy 2 & 0.1986 & 1.31 & no \\
\hline 2 FGLJ1627.8+3219 & $\mathrm{J} 162800.39+322414.0$ & QSO & 0.9051 & 0.69 & yes \\
\hline 1FGLJ2117.8+0016 & J211817.39+001316.7 & Sy 1.5 & 0.4629 & 0.79 & no \\
\hline
\end{tabular}

Col. (1) Source name.

Col. (3) SDSS spectroscopic classification based on our analysis.

Col. (4) Redshift estimate derived from our analysis. Question mark indicates uncertain estimates.

$(+)$ : 2FGLJ2223.4+0104 is the source indicated by Cowperthwaite et al. (2013).

Col. (6) Classification flag: (yes) marks sources that have been classified on the basis of our analysis. 
TABLE 4

SUMMARY

\begin{tabular}{|lrrrrr|}
\hline Sample & Tot. & BZBs & BZQs & candidates & new $z$ \\
\hline$\left(1^{\text {st }}\right):$ ROMA-BZCAT & 84 & $47(47)$ & $3(3)$ & $34(20)$ & 18 \\
$\left(2^{\text {nd }}\right): \gamma$-ray blazar candidates & 27 & $7(7)$ & $8(7)$ & $12(4)$ & 4 \\
\hline
\end{tabular}

Col. (2) Total umber of sources listed in the sample.

Col. (3) Number of sources classified as BZBs; those confirmed by our analysis are in parenthesis.

Col. (4) Number of sources classified as BZQs; those confirmed by our analysis are in parenthesis.

Col. (5) Number of blazar candidates; those confirmed by our analysis are in parenthesis.

Col. (6) Sources with new $z$ estimates. 